\title{
Northeastern Asian
}

National Cancer Institute

\section{Source}

National Cancer Institute. Northeastern Asian. NCI Thesaurus. Code C153342.

Denotes a person whose ancestry is in any of the Asian countries bordering the Pacific Ocean: China, Japan, Mongolia, North Korea, and South Korea. 